\title{
Optimized chromatographic conditions for separation of halogenated acetic acids by ultra-performance liquid chromatography-electrospray ionization-mass spectrometry
}

\author{
Qian Luo, Donghong Wang, Zi Wei, Zijian Wang* \\ State Key Laboratory of Environmental Aquatic Chemistry, Research Center for Eco-Environmental Science, Chinese Academy of Sciences, Beijing 100085, China
}

\section{A R T I C L E I N F O}

\section{Article history:}

Received 10 October 2012

Received in revised form

16 December 2012

Accepted 20 December 2012

Available online 1 January 2013

\section{Keywords:}

Iodoacetic acids

Mixed halogenated acetic acids

Disinfection by-products

Drinking water

Volatile ion pair reagent

Ultra-performance liquid chromatography

column

\begin{abstract}
A B S T R A C T
Emerging halogenated acetic acids (HAAs), especially mixed halogenated acids such as chlorobromo, chloroiodo- and bromoiodo-acetic acids, are unregulated disinfection by-products in drinking water. Because these compounds are hydrophilic and strongly acidic, they are difficult to detect at trace levels using approved analytical methods. In the present study, 13 HAAs were effectively separated on three ultra-performance liquid chromatography columns. The effects of changing in the aqueous mobile phase, acidic solutions and cationic volatile ion pair reagents were investigated. The samples were pretreated by filtration, and extraction, while derivatization and concentration procedures were not required. The limits of quantitation for regulated HAAs were between $0.5 \mu \mathrm{g} / \mathrm{L}$ and $1.7 \mu \mathrm{g} / \mathrm{L}$ and for unregulated HAAs were 1.2 and $5.8 \mu \mathrm{g} / \mathrm{L}$, especially for the iodinated acetic acids were 1.5 and $2.1 \mu \mathrm{g} / \mathrm{L}$. The method was applied to two finished water samples collected in China (Shanghai and Xuzhou) from water treatment plants that use chlorine for disinfection. Multiple unregulated HAAs were found in the two samples, but iodoacids were only detected in the water sample from Shanghai, which could be attributed to the characteristics of the source water. The presence of unregulated HAAs, especially mixed bromo- and iodoacetic acids, in the finished water samples could affect human health, and this warrants further investigation.
\end{abstract}

(C) 2012 Elsevier B.V. All rights reserved.

\section{Introduction}

Halogenated acetic acids, especially mixed bromo- and iodoacetic acids, are of public health concern as emerging disinfection by-products (DBPs) in drinking water. Chloroacetic acids have various health effects, with dichloroacetic acid (DCAA) and trichloroacetic acid (TCAA) causing severe skin and eye irritation in humans at high concentrations and liver tumors in mice exposed to drinking water containing these two chemicals [1]. The Integrated Risk Information System classifies DCAA as a probable human carcinogen (Group B2) and TCAA as a possible human carcinogen (Group C) [2,3]. The World Health Organization and China have established maximum contamination levels (MCLs) of $50 \mu \mathrm{g} / \mathrm{L}$ for DCAA and $100 \mu \mathrm{g} / \mathrm{L}$ for TCAA [4,5]. Chloroacetic acids are formed from dissolved humic matter during disinfection of drinking water with chlorine. Bromoacetic acids are also formed in drinking water during disinfection, and these compounds are included as DBPs. Conversion of chlorinated acetic acids to brominated ones often occurs when bromide levels in the source waters

\footnotetext{
* Corresponding author. Tel.: +86 10 62849140; fax: +861062849140.

E-mail address: wangzj@rcees.ac.cn (Z. Wang).
}

are high. DCAA, TCAA, monochloroacetic acid (MCAA), monobromoacetic acid (MBAA) and dibromoacetic acid (DBAA) are regulated under the Stage 1 and 2 D/DBP Rule by the US Environmental Protection Agency (EPA), and MCLs of $60 \mu \mathrm{g} / \mathrm{L}$ have been established for the sum of these five types of HAAs (HAA5) [6]. The HAA5 along with four unregulated mixed bromochloro-acetic acids including tribromoacetic acid (TBAA), bromochloroacetic acid (BCAA), dichlorobromoacetic acid (DCBAA) and dibromochloroacetic acid (DBCAA) are known as HAA9. In recent years, more species of these HAAs have been frequently found in disinfected drinking water, and this could affect human health. Tap water from 10 locations in Taiwan was found to contain 1-13 $\mu \mathrm{g} / \mathrm{L}$ (individual concentrations) of six species of HAAs with total concentrations ranging from $5 \mu \mathrm{g} / \mathrm{L}$ to $33 \mu \mathrm{g} / \mathrm{L}$ [7]. HAA9 concentrations in plant effluent from 12 drinking water treatment plants in the US ranged from $5 \mu \mathrm{g} / \mathrm{L}$ to $34 \mu \mathrm{g} / \mathrm{L}$ [8]. Tap water samples collected from five water treatment plants in Shandong, China had concentrations of HAA9 from $7.79 \mu \mathrm{g} / \mathrm{L}$ to $36.5 \mu \mathrm{g} / \mathrm{L}$ [9]. Additionally, a recent nationwide investigation in the US showed higher concentrations for brominated acetic acids than those of the chlorinated species [8,10]. Because the source waters for these drinking waters have high bromide/iodide and total organic carbon (TOC) levels, it is likely that the concentrations are in the upper range for potential levels of bromo- and 
iodoacetic acids. Monoiodoacetic acids (MIAA) were first reported in a survey of finished drinking water in 2006 [8]. A recent study showed that MIAA and chloroiodoacetic acids (CIAA) are formed when chlorinated tap water was boiled or stored for a long time with iodized table salt [11]. Among the DBPs that have been found in disinfected drinking water, iodinated acetic acids are the most concerning because of their higher cytotoxicity and genotoxicity compared with the corresponding chloro- and bromo-acetic acids [12,13]. Iodoacids toxicity can be ranked for their toxicity as follows: MIAA $\gg$ diiodoacetic acid (DIAA) > bromoiodoacetic acid (BIAA) [12]. Iodinated DBPs in drinking water are generally believed to form in the chlorine and chloramination disinfection process because of natural iodide in the source waters [14]. MIAA is formed predominantly and detected more often than the other iodoacids in disinfected water [15]. There are other bromo- and iodoacetic acids that are unregulated and have not been of concern till now. Consequently analytical methods are not available for these compounds.

Measurement of HAAs in drinking water is challenging, especially at low levels, because these compounds are strongly hydrophilic and acidic. To date, a series of methods established by the US EPA have been used for compliance monitoring of the HAA5 analogs, and especially for DCAA and TCAA in disinfected water. The acids are generally derivatized to their corresponding methyl esters using acidic methanol or diazomethane before analysis by gas chromatography with electron capture detection or mass spectrometry (MS) [16]. Liquid chromatography (LC)-MS has been applied to HAAs analysis in drinking water at the $\mu \mathrm{g} / \mathrm{L}$ level. However, the most popular stationary phases in LC columns do not perform well for retention of small and polar compounds such as DCAA and TCAA. In theory, highly polar compounds require efficient ion-pairing to obtain reasonable retention. Ion chromatography (IC) with a post-column suppressor to decrease the interference of matrices with very high ionic strength has been coupled with electrospray ionization (ESI)-MS to obtain sub- $\mu \mathrm{g} / \mathrm{L}$ sensitivity for the HAA5 species [17,18]. A validated LC-MS/MS method using ion-exchange hydrophilic interaction chromatography (HILIC) was used to analyze DCAA at levels as low as $1 \mu \mathrm{g} / \mathrm{L}$, but this method is not useful for other HAAs [19]. Although DCAA could be effectively retained on an amino column for HILIC chromatography, this technique failed to separate the HAA9 [20]. This method also cannot be used for fast and direct analysis because the concentrated water sample of HAAs needs to be dissolved in an organic mobile phase with low water content. Ultra-performance LC (UPLC)-MS has been used to measure the HAA9 in drinking water using HILIC and BetaMax Acid columns after 40- and 400-fold enrichment [21] and a C8 column without enrichment [9]. In these studies LODs at $\mu \mathrm{g} / \mathrm{L}$ levels were obtained. Thus, volatile ion pair reagents that are aliphatic could be suitable for retention of the HAA5 in LC-ESI-MS [13,22]. Traditionally, iodoacids are analyzed by GC/MS after derivation because iodinated DBPs are highly polar [12]. Recently they have been analyzed by LC/MS using a modified column (porous graphitic carbon) for retention of MIAA [23]. To improve the sensitivity, large volume direct aqueous injection with LC/ESI/MS/MS was applied for the analysis of four iodo-HAAs (MIAA, CIAA, BIAA, and DIAA) at low concentration (ng/L) in drinking water [24]. Optimization of UPLC/MS methods would be a logical choice for emerging mixed HAAs in disinfected water, and especially for more hydrophilic acidic DBPs.

In this study, we present a comprehensive and effective analytical method for simultaneous analysis of underivatized HAAs based on UPLC-ESI-MS/MS. The chromatographic conditions and MS parameters were optimized for analysis of unregulated and regulated HAAs. Different commercial UPLC columns, acetic mobile phases, and volatile ion pair reagents were investigated to obtain better retention and resolution for the HAAs, especially for bromo-, iodo- and mixed HAAs. The UPLC-ESI-MS/MS method was applied to drinking water samples from two cities in China.

\section{Material and methods}

\subsection{Chemicals and reagents}

A mixed standard solutions of HAAs (MCAA, DCAA, TCAA, MBAA, DBAA, BCAA, TBAA, CDBAA and DCBAA) with concentrations between $20 \mathrm{mg} / \mathrm{L}$ and $200 \mathrm{mg} / \mathrm{L}$ in methyl tert-butyl ether, was obtained from AccuStandard (New Haven, CT). The qualitative, quantitative, and recovery internal standards were bromoacetic acid $\left({ }^{13} \mathrm{C}_{1}, 99 \%\right)$ and chloroacetic acid $\left({ }^{13} \mathrm{C}_{2}, 99 \%\right)$ from Carmbridge Isotope Laboratories (Andover, MA). MIAA ( $\geq 99.0 \%$ ) was purchased from Sigma-Aldrich (St. Louis, MO). Chloroiodoacetic acid (CIAA, $\geq 90 \%$ ), BIAA ( $\geq 85 \%$ ), and DIAA ( $\geq 90 \%$ ) were purchased from Synthesis Homes Inc. (Toronto, Canada). A stock solution $(200 \mathrm{mg} / \mathrm{L}$ in methanol) was prepared using MIAA, CIAA, BIAA and DIAA standards. An intermediary standard solution $(20 \mathrm{mg} / \mathrm{L})$ containing a mixture of the twelve HAAs was prepared by diluting the standard solution in methanol/water $(1: 1, v / v)$. Working solutions were prepared daily by diluting the intermediary standard solution with distilled water (Milli-Q water). Ammonium acetate (>99\%), ammonium formate ( $>99 \%$ ), formic acid ( $\geq 99 \%$ ) and acetic acid ( $\geq 99.7 \%$ ) (mass spectrometry grade) and L-ascorbic acid (analytical reagent grade) were purchased from Sigma-Aldrich. Four volatile ion pair reagents, including triethylamine (TEA) (99.5\%), tributylamine (TBA), dibutylamine (DBA) and dimethyl- $n$-butylamine (DMBA) (98\%) were purchased from TCI America (Portland, OR). Chemical reagents, including acetonitrile $(A C N)$ and methanol, were of LC-MS grade and obtained from Thermo Fisher Scientific (Waltham, MA). All standard solutions were stored at $4{ }^{\circ} \mathrm{C}$. Distilled water was prepared using a Milli-Q system (Millipore, Billerica, MA) and used to dilute the mobile phase and solutions, and as a blank. A PSF GHP membrane filter needle $(13 \mathrm{~mm} \times 0.2 \mu \mathrm{m})$ from Pall Corporation (Port Washington, NY) was used for sample purification.

\subsection{Sample preparation}

The two finished water samples were collected between 2009 and 2010 from water treatment plants in China that use chlorine for disinfection. L-Ascorbic acid was added to the water samples at a concentration of $20 \mathrm{mg} / \mathrm{L}$ to remove residual chlorine. Water samples with a surrogate standard $\left({ }^{13} \mathrm{C}_{1}\right.$-MBAA) were transported immediately to our laboratory in glass containers under cool and dark conditions. The samples were analyzed within 1 day of collection. Each water sample was filtered through a $0.2 \mu \mathrm{m}$ membrane filter needle and injected into the UPLC-MS/MS for analysis. An aliquot $(990 \mu \mathrm{L})$ of the standard solution or sample was filtered through a $0.2 \mu \mathrm{m}$ GHP membrane filter and transferred to a glass vial. The ${ }^{13} \mathrm{C}_{2}$-MCAA standard $(10 \mu \mathrm{L}, 5 \mathrm{mg} / \mathrm{L})$ was added to the standard solution or sample before UPLC-MS/MS analysis.

\subsection{UPLC-MS/MS analysis}

The UPLC analyses were performed using a Waters Acquity Ultra Performance LC system (Waters, Milford, MA). UPLC separation was achieved using Acquity UPLC ${ }^{\mathrm{TM}}$ HSS T3 column $(2.1 \mathrm{~mm} \times 100 \mathrm{~mm}$; $1.8 \mu \mathrm{m})$, HSS C18 column $(2.1 \mathrm{~mm} \times 100 \mathrm{~mm} ; 1.8 \mu \mathrm{m})$ and BEH C18 column $(2.1 \mathrm{~mm} \times 100 \mathrm{~mm} ; 1.7 \mu \mathrm{m})$ from Waters Corporation. The column temperature was $40^{\circ} \mathrm{C}$, and the mobile phase flow rate was $0.3 \mathrm{~mL} / \mathrm{min}$. The sample volume injected was $10 \mu \mathrm{L}$. The mobile phase was composed of solvent $A$, which contained water, formic acid, acetic acid, formic ammonium, acetic ammonium buffer, and $5-10 \mathrm{mmol} / \mathrm{L}$ of the ion pair regent (EA, TBA, DBA and DMBA), and solvent $B(100 \% A C N)$. The solvent gradient program for the 
Table 1

Optimized multiple reaction monitoring conditions and MS parameters for analysis of target HAAs.

\begin{tabular}{|c|c|c|c|c|c|}
\hline & Precursor ion $(m / z)$ & Product ion $(m / z)$ & Dwell (S) & Cone voltage $(V)$ & Collision energy $(V)$ \\
\hline MCAA & 92.6 & 34.8 & 0.1 & 20 & 10 \\
\hline${ }^{13} \mathrm{C}_{2}$-MCAA & 93.9 & 34.8 & 0.1 & 20 & 10 \\
\hline MBAA & 136.8 & 78.9 & 0.05 & 12 & 15 \\
\hline${ }^{13} \mathrm{C}_{1}-\mathrm{MBAA}$ & 140.2 & 80.9 & 0.05 & 12 & 20 \\
\hline MIAA & 184.9 & 126.8 & 0.05 & 10 & 10 \\
\hline DCAA & 126.6 & 82.6 & 0.05 & 12 & 10 \\
\hline BCAA & 172.8 & 128.7 & 0.05 & 20 & 15 \\
\hline DBAA & 216.8 & 173 & 0.05 & 20 & 20 \\
\hline CIAA & 218.8 & 127.1 & 0.1 & 25 & 8 \\
\hline BIAA & 265.4 & 127.1 & 0.1 & 30 & 30 \\
\hline DIAA & 267.7 & 127.1 & 0.1 & 30 & 20 \\
\hline TCAA & 160.8 & 116.8 & 0.1 & 15 & 8 \\
\hline BDCAA & 206.7 & 162.8 & 0.1 & 20 & 10 \\
\hline CDBAA & 250.8 & 206.7 & 0.1 & 15 & 7 \\
\hline TBAA & 248.8 & 78.9 & 0.1 & 20 & 20 \\
\hline
\end{tabular}

BEH C18 and HSS C18 columns was as follows: 5\% solvent B for $0.1 \mathrm{~min}$; increased to $60 \%$ solvent B over $4 \mathrm{~min}$; increased to $100 \%$ solvent B over $1 \mathrm{~min}$; reduced to $20 \%$ solvent B over $1.0 \mathrm{~min}$; and then re-equilibrated for $1.5 \mathrm{~min}$ before the next sample injection. The solvent gradient program for the HSS T3 column began with $0 \%$ solvent $\mathrm{B}$, but otherwise followed the same steps described above.

Determination was performed using a Waters Micromass Quattro Premier XE tandem quadrupole MS (Waters, Milford, MA). The instrument was operated using an electrospray source in negative mode. The ionization source parameters were as follows: capillary voltage, $3.0 \mathrm{kV}$; source temperature, $120^{\circ} \mathrm{C}$; desolvation gas $\left(\mathrm{N}_{2}\right)$ temperature, $380^{\circ} \mathrm{C}$; desolvation gas flow rate, $600 \mathrm{~L} / \mathrm{h}$; and cone gas flow rate, $50 \mathrm{~L} / \mathrm{h}$. Nitrogen (99.9\% pure) and argon (99.9999\% pure) were used as the cone and collision gases, respectively. Flow injection analysis was performed to obtain the precursor and product ion, the cone voltage, and collision energy for each compound. Multiple reaction monitoring (MRM) transitions and the individual cone voltage and collision energy voltages applied for the analyses are summarized in Table 1 . The inter-scan delay was set at $10 \mathrm{~ms}$. Data acquisition was carried out by MassLynx V 4.1 software.

\section{Results and discussion}

\subsection{Optimizing multiple reaction monitoring conditions in MS}

In general, the sensitivity for target compounds in MS varies according to the infusion concentration and MS parameters. ESI mode was used to detect the anionic HAAs because greater insource fragmentation of the cations was observed when using atmospheric pressure chemical ionization $[25,26]$. Determining the ESI parameters of different halogenated species of HAAs is critical because the acidic mobile phase may affect the degree of ionization of analytes and/or formation of complexes between the ion pair reagents and the HAAs, and this could influence signal intensities. MRM mode in MS/MS was used to obtain high sensitivity and specificity for the HAAs in this study. With ESI in negative mode three types of precursor ions for the HAAs, $[\mathrm{M}-\mathrm{H}]^{-}$(pseudomolecular ion), $[\mathrm{M}-\mathrm{COOH}]^{-}$(decarboxylated ion), and $[2 \mathrm{M}-\mathrm{H}]^{-}$ (dimer ion) were detected. Chlorinated acetic acids are apt to form deprotonated $[\mathrm{M}-\mathrm{H}]^{-}$to produce higher signal intensities in the MS. However, in this study, dimer ions were the major precursor ions of MCAA, DCAA and TCAA at relative high infusion concentrations $(>1 \mu \mathrm{g} / \mathrm{L})$ and pseudomolecular ions became predominant at low concentrations, which is similar to the results in a previous study [27].For the five HAAs (MCAA, DCAA, MBAA, BCAA and DBAA) and two ${ }^{13} \mathrm{C}$ labeled HAAs (13 $\mathrm{C}_{1}$-MBAA and $13 \mathrm{C}_{2}$-MCAA), $[\mathrm{M}-\mathrm{H}]^{-}$was selected as the precursor ion and $[\mathrm{M}-\mathrm{COOH}]^{-}$as the product ion. The degree of halogen substitution influenced the patterns of HAAs ions formed in ESI, especially forBDCAA, CDBAA and TBAA, and the relative signal intensities of $[\mathrm{M}-\mathrm{COOH}]^{-}$increased. The sensitivity was higher for deprotonated ions of TBAA in MRM than selected ion monitoring mode, and we found $[\mathrm{M}-\mathrm{COOH}]^{-}$ was more favored in ESI than $[\mathrm{M}-\mathrm{H}]^{-}$for more heavily substituted HAAs. Different ESI probes and operating conditions may cause differences in the relative intensities of base ions. In the present study, the optimized precursor ions for CDBAA and BDCAA were $[\mathrm{M}-\mathrm{H}]^{-}$ and $[\mathrm{M}-\mathrm{COOH}]^{-}$, respectively. The precursor ions of MIAA, CIAA, and BIAA were $[\mathrm{M}-\mathrm{H}]^{-}$ions, in a similar manner to the monoand di-chlorinated and brominated substitutes. Additionally, the product of the three iodinated acids was the iodide ion. For DIAA, the precursor ion was $[\mathrm{M}-\mathrm{COO}]^{-}$and the product ion was iodine. These results indicate that highly brominated and iodinated HAAs (TBAA, CIAA, BIAA and DIAA) are likely to form halogen ions as the product ion.

The main operating parameters that affect the performance of ESI are the cone voltage (capillary exit voltage) and collision energy. In this experiment, one of these parameters was fixed while another was optimized for each of the HAAs. The cone voltage is applied to the capillary and affects the transmission and fragmentation of sample ions. Generally, more fragmentation will occur as the cone voltage increases. In addition, an acidic mobile phase can increase the retention time of the analytes but suppress the signal intensity, and the extent of this is dependent on the individual species [28]. To eliminate the influence of amines (volatile ion pair reagents), a higher fragmentor voltage (40 V) was used in an earlier study [29]. In this study, the optimum cone voltage was evaluated for each of the compounds. The intensities of the deprotonated ions and the decarboxylated ions for all HAAs are shown as a function of the cone voltage. For the monohaloacetic acids (MCAA, MBAA and MIAA) and dihaloacetic acids (DCAA, DBAA, BCAA, CIAA and BIAA), the deprotonated ion was observed as the base peak at less than $30 \mathrm{~V}$ and the sensitivity was maximized. Thus, the cone voltages of the chloroand bromoacetic acids were less than those of the iodinated ones. At higher cone voltages, the sensitivity for the deprotonated ion decreased and the sensitivity for the decarboxylated ion increased. This was similar for DIAA and TBAA, where the sensitivities for the deprotonated ions were very low and the decarboxylated ions were observed as the base peaks at 20-30 V. The maximum sensitivities for the base peaks for all HAAs were obtained at 15-30 V. The capillary cone and collision voltage conditions for the 13 HAAs are listed in Table 1. From the above results, the collision energy for each HAA was set at a different voltage. In the typical mass spectra, the collision energy of the chlorinated and brominated acetic acids were $1<20 \mathrm{~V}$ and that of the di-iodinated acetic acids was $>20 \mathrm{~V}$ (Table 1). 
3.2. Separation and retention of HAAs on column with acidic mobile phase

The chromatography column and mobile phase are the most important parameters for a separation method for target compounds. HAAs are generally difficult to separate using LC columns because they are hydrophilic and strongly acidic with $p K a$ values ranging from 0.63 to $4.1[28,30]$. The major commercially available UPLC columns are the HSS series (High Strength Silica; T3 and $\mathrm{C} 18$ ) and the BEH series (Ethylene Bridged Hybrid; C8 and C18), which have slightly different solid phases to routine LC columns. Our experimental results confirmed that a conventional C18 column (Fig. S1) performed poorly for retention of HAAs because of its nonpolar stationary phase [27]. All the HAAs were not efficiently separated and had similar retention times on the BEH C18 column, even though the BEH particle is a bonded phase for reversedphase and hydrophilic interaction chromatography. This meant these compounds eluted in the dead volume and did not separate (Table 2). Recent research indicated that a BEH C8 column could separate nine HAAs with water as the mobile phase [9]. However, similar results were not obtained in the present study for the HAAs on a similar BEH C18 column. Successful separation of HAAs using a C8 column could be attributed to hydrophilic affinity with the stationary phase of C8 [9]. However, the C18 column should theoretically provide better retention of HAAs than a C 8 column because longer chain lengths will be more appropriate for retention of small hydrophilic molecules. Therefore, we compared columns and found the HSS C18 column captured HAAs better than a BEH C18 column. The separation principles with the HSS C18 column are slightly different to the BEH C18 column because the HSS C18 stationary phase has a tri-functionally bonded C18 ligand and proprietary endcapping process that yields superior peak shapes in mobile phases with low $\mathrm{pH}$ values. The target HAAs were effectively separated on the HSS C18 column with water, acid, and acidic buffer as the aqueous mobile phase (Table 2 ).

In previous studies, aqueous acidic solutions have been used as the mobile phase in HAAs analysis to reduce dissociation of the HAAs and to improve their retention and separation on LC columns [31,32]. Ammonium acetate buffer could retain HAAs on a BetaMax acid column but water could not [19]. Therefore, $0.1 \%$ formic acid, $0.1 \%$ acetic acid, $5 \mathrm{mmol} / \mathrm{L}$ ammonium formate-formic acid buffer ( $\mathrm{pH} 3.5-4.0$ ), and $5 \mathrm{mmol} / \mathrm{L}$ ammonium acetate-acetic acid buffer ( $\mathrm{pH} 4.0-4.5$ ) were investigated as mobile phases to obtain the most effective separation of the target HAAs. Because the $\mathrm{pKa}$ values of the 13 HAAs are very low, most of the analytes should exist in their ionic forms at $\mathrm{pH} 4.1[28,30]$. With formic or acetic acid as the mobile phase, retention of the HAAs increased compared with when ammonium formate or ammonium acetate buffer was used as the mobile phase (Table 2). This may be because of the increased strength of the mobile phase with the increased content of formic acid or acetic acid. Alternatively, this could be attributed to decreased silanol activity and reduced interaction between the analytes and the stationary phase. Acid modifies the interactions between the analytes and the two phases to improve the chromatographic peak shape, or simply acts as a source of protons [33]. In general, the retention of most the HAAs varied according to their octanol-water partition coefficients, expect for TCAA, which was not affected by the $\mathrm{pH}$ value of the mobile phase. Retention of dihalogenated acids (DCAA, BCAA, DBAA, CIAA, BIAA and DIAA) on the column was stronger with formic and acetic acid mobile phases than with water and acidic buffers. The HSS T3 bonding utilizes a trifunctional $\mathrm{C} 18$ alkyl phase bonded at a ligand density that promotes polar compound retention and aqueous mobile-phase compatibility. Formic acid gave the best retention and separation of the 10 HAAs in the water samples [28]. The T3 end-capping was much more effective than traditional trimethyl silane end-capping and

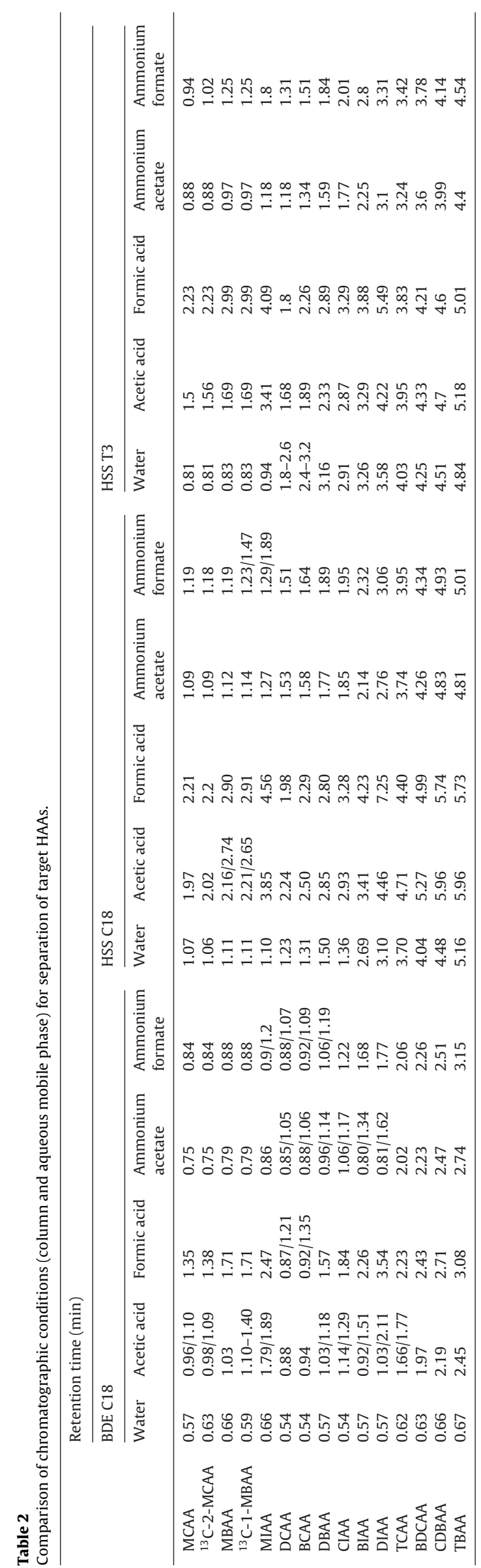


provided superior polar compound retention and aqueous compatibility. In this study, the HSS T3 column was used to analyze HAAs with different aqueous mobile phases, including formic acid. High retention on $\mathrm{T} 3$ resulted in increased elution of the HAAs with mobile phases containing higher aqueous fractions (elution with $100 \%$ aqueous mobile phase). The HSS T3 column was used to optimize chromatographic separation of the 13 HAAs, and retention of these compounds was better than on a HSS C18 column (Table 2). A high percentage of water $(>99 \%)$ gave very low starting eluent strength and provided better retention for the analytes.

An analyte tends to diffuse in the mobile and stationary phases while moving down the column, which may cause peak broadening. This was observed for DCAA and BCAA on the T3 column with acetic acid as the mobile phase. This observation indicates predominance of van der Waals and/or dispersion interactions between the analytes and the stationary phase in the elution process [34]. Simultaneously, water as a highly polar eluent will carry the ions away quickly. Addition of acid or acidic buffer solutions to the mobile phase could increase retention of the HAAs.

\subsection{Influence of the volatile ion pair reagent on the retention of HAAs}

All 13 target HAAs were not separated in the BEH C18 column with water as the mobile phase (Fig. S1). However, they could be separated with addition of a volatile ion pair reagent. The volatile ion pair reagent enhanced retention and improved the peak shape. TEA has consequently been used as an ion pair reagent in several applications for LC-MS because it does not interfere with MS detection in the concentrations normally applied for HPLC [19,21]. The properties (type and chain length of the hydrophobic group) of the ion pair reagent are important for retention on the UPLC column. Therefore, four ion pair reagents were tested, including TEA, TBA, DBA and DMBA. The acetic acid ion generally exchanges with the counter ion of the ion-pair reagent, which increases interaction with the column and improves retention of the sample. Retention behavior generally reflects both the strength of the ion pair reagent and the strength of the interaction with different types of analytes. In this study, highly polar compounds, such as MCAA, MBAA and DCAA, required more efficient ion pair reagents to obtain reasonable retention. The retention behaviors obtained with different concentrations of these amines, and the influence on the sensitivity, were studied for 13 of the HAAs. By comparing the separation and sensitivity results for the 13 HAAs using the four volatile amines, optimized conditions were obtained for the different UPLC columns. All amines provided sufficient retention for dihaloacetic acids and trihaloacetic acids (Table 3 ). However, the more polar monohaloacetic acids (MCAA and MBAA) were poorly retained with DMBA. With TBA, monohaloacetic acids were retained strongly, but separation of MCAA and MBAA was not improved and TBAA could not be detected. The pKa values of TEA and DBA are 10.75 and 11.25, respectively.

Sensitivity in ESI-MS is dependent on the composition of the mobile phase, including the concentration, type of ion pair reagent, and the $\mathrm{pH}$ value. In this study, the peak areas of the HAAs tended to decrease with increasing amine concentration from $0 \mathrm{mmol} / \mathrm{L}$ to $5 \mathrm{mmol} / \mathrm{L}$ ion pair reagent. This decrease appeared to be caused by formation of cluster ions with anionic analytes. To obtain maximum sensitivity, the concentration of the ion pair reagent should be kept as low as possible for sufficient chromatographic retention [35]. The influence of the mobile phase $\mathrm{pH}$ on the ion formation was studied for DBA only because the type of amine does not affect ion formation. The ion intensities using DBA as the ion pair regent did not change for seven of the HAAs between $5 \mathrm{mmol} / \mathrm{L}$ and $20 \mathrm{mmol} / \mathrm{L}$. The exceptions were DCAA, TBAA and MIAA. The intensities of all the HAAs reached their maxima with $1 \mathrm{mmol} / \mathrm{L}$ DBA. Despite the general trend of decreasing intensity with increasing amine concentration, an increase in ion intensity was found with increasing DBA concentration from $0.5 \mathrm{mmol} / \mathrm{L}$ to $5 \mathrm{mmol} / \mathrm{L}$ for all target HAAs. Because the sensitivity deceases with increasing amine concentrations in the UPLC effluent, low concentrations of the ion-pairing agent are desirable. DBA provided the best retention for the separation at low amine concentrations with many different UPLC columns, such as the HSS T3 column, and was chosen as the ion pair reagent for these compounds (Fig. 1). TEA as ion-pairing agent gave effective separation, especially for few pair of analytes (such as CDBAA and TBAA) (Fig. S2). To avoid formation of ion pairs with cationic analytes that would appear neutral to the electrospray process, an acidic aqueous mobile phase was used in this study. Water containing formic acid, acetic acid, or ammonium acetate buffer ( $\mathrm{pH}$ value: 4-7) was investigated as the mobile phase. Finally, water containing $0.1 \%$ formic acid was selected as the mobile phase because of the relatively good separation and high sensitivity it provided for most of the HAAs. It is interesting that MCAA was retained well on the HSS C18 column with many different mobile phases, expect for DMBA and TEA (Fig. 2). Good retention of the iodoacids (such as MIAA in Fig. 3) with $0.1 \%$ formic acid and all four volatile ion pair reagents was obtained.

\subsection{Linearity, detection limits and precision of the UPLC-MS/MS}

The brominated acetic acids (especially MBAA) can decompose at high temperatures and $\mathrm{pH}$ values. Therefore, ${ }^{13} \mathrm{C}_{1}$-MBAA was used as a stable alternative for accurate tracking of the MBAA analyte. The stable labeled internal standard was ${ }^{13} \mathrm{C}_{2}$-MCAA. The relative response factors (RRFs) for the 13 target HAAs and the surrogate standard ( ${ }^{13} \mathrm{C}_{1}$-MBAA) were calculated based on the ratio of the relative peak area of each analyte to that of the internal standard $\left({ }^{13} \mathrm{C}_{2}\right.$-MCAA), which was automatically obtained by TargetLynx software. The reproducibility of the RRFs was also determined. Quality control measures included injection of a blank solution (the mobile phase) to check for carryover after each sample, and analysis of a set of standard solutions to calculate the RRF of each compound before and after analysis of the authentic drinking water samples. The linear relationship, limit of detection (LOD) and limit of quantitation (LOQ) are shown in Table 4. The average $(n=6)$ concentration of the 13 target HAAs in control samples containing standards $(10 \mu \mathrm{g} / \mathrm{L})$ was $9.78 \mu \mathrm{g} / \mathrm{L}(\mathrm{RSD}=2.2 \%)$.

To achieve optimum sensitivity, all experiments were carried out under the optimized UPLC and MS/MS conditions. Calibration curves were constructed over the concentration range $0.1-100 \mu \mathrm{g} / \mathrm{L}$. The calibration curves of the chloro- and bromoacetic acids were linear with good correlation coefficients (0.9754-0.9999), and the correlation coefficient for MIAA was 0.9213 (Table 4$)$. The LODs $(n=3)$ of the 13 HAAs were between $0.15 \mu \mathrm{g} / \mathrm{L}$ and $1.5 \mu \mathrm{g} / \mathrm{L}$, and their LOQs $(n=3)$ were between $0.5 \mu \mathrm{g} / \mathrm{L}$ and $5.8 \mu \mathrm{g} / \mathrm{L}$. It should be noted that LOD and LOQ of mixed halogenated acetic acids, such as BDCAA and CDBAA, were higher than those of the tri-halogenated acetic acids (TBAA and TCAA). The mean recoveries $(n=6)$ of 13 HAAs in the spiked water samples $(10 \mu \mathrm{g} / \mathrm{L})$ were between $85 \%$ and $110 \%$, and the RSD were all less than $8.7 \%$.

\subsection{Occurrence and concentration of HAAs in finished water from China}

The optimized method was applied to two drinking water samples from Shanghai and Xuzhou, China (anion concentrations shown in Table S1). In general, co-existing anions (particularly chloride and sulfate) at high concentrations could interference with the ESI/MS sensitivity if injected directly. The anion concentrations in the water samples collected from P1 (Table S1) were lower 
Table 3

Comparison of chromatographic conditions (column and aqueous mobile phase) for separation of target HAAs.

\begin{tabular}{|c|c|c|c|c|c|c|c|c|c|c|c|c|}
\hline & \multicolumn{12}{|c|}{ Retention time (min) } \\
\hline & \multicolumn{4}{|l|}{ BEH C18 } & \multicolumn{4}{|l|}{ HSS C18 } & \multicolumn{4}{|l|}{ HSS T3 } \\
\hline & TEA & BDEA & DBA & TBA & TEA & BDEA & DBA & TBA & TEA & BDEA & DBA & TBA \\
\hline MCAA & $0.88 / 1.09$ & 1.15 & 1.68 & 2.19 & $1.15 / 1.55$ & $1.28 / 1.76$ & 3.00 & 3.76 & $1.66-1.93$ & $1.97 / 2.63$ & 3.41 & 3.42 \\
\hline${ }^{13} \mathrm{C}-2-\mathrm{MCAA}$ & $0.84 / 1.07$ & 1.14 & 1.70 & 2.20 & 1.54 & 1.73 & 2.89 & 4.20 & 1.93 & 2.64 & 3.39 & 3.42 \\
\hline MBAA & $0.89 / 1.11$ & 1.23 & 1.79 & 2.39 & $1.19 / 1.60$ & $1.31 / 1.97$ & 3.24 & 4.07 & $2.22-2.45$ & 2.96 & 3.44 & 3.55 \\
\hline${ }^{13} \mathrm{C}-1-\mathrm{MBAA}$ & $0.86 / 1.07$ & 1.23 & 1.86 & 2.45 & 1.59 & 1.93 & 3.08 & 4.44 & 2.43 & 2.93 & 3.44 & 3.57 \\
\hline MIAA & 1.20 & 1.40 & 2.06 & 2.80 & 1.85 & 2.23 & 3.38 & 4.81 & 2.95 & 3.18 & 3.52 & 3.61 \\
\hline DCAA & 1.53 & 1.75 & 2.42 & 3.53 & 2.38 & 2.82 & 4.29 & 5.44 & 3.24 & 3.42 & 3.83 & 3.81 \\
\hline BCAA & 1.67 & 1.90 & 2.58 & 3.78 & 2.61 & 3.08 & 4.55 & 5.63 & 3.39 & 3.59 & 3.94 & 3.87 \\
\hline DBAA & 1.89 & 2.14 & 2.80 & 4.04 & 2.94 & 3.42 & 4.86 & 5.89 & 3.63 & 3.82 & 4.09 & 3.95 \\
\hline CIAA & 1.83 & 2.22 & 2.88 & 4.54 & 2.99 & 3.46 & 4.64 & 6.15 & 3.60 & 3.89 & 4.07 & 4.07 \\
\hline BIAA & 2.19 & 2.51 & 3.16 & 4.98 & 3.38 & 3.88 & 5.02 & 6.47 & 3.89 & 4.19 & 4.15 & 4.17 \\
\hline DIAA & 2.66 & 3.01 & 3.56 & 5.47 & 4.04 & 4.54 & 5.56 & 6.93 & 4.36 & 4.70 & 4.59 & loss \\
\hline TCAA & 3.35 & 3.73 & 4.21 & 5.73 & 5.00 & 5.51 & 6.36 & 7.42 & 5.06 & 5.38 & 4.99 & 4.65 \\
\hline BDCAA & 3.61 & 3.99 & 4.42 & 5.93 & 5.31 & 5.82 & 6.59 & 7.36 & 5.30 & 5.60 & 5.13 & 4.77 \\
\hline CDBAA & 3.90 & 4.27 & 4.64 & 6.17 & 5.66 & 6.15 & 6.85 & 7.35 & 5.56 & 5.86 & 5.28 & 4.88 \\
\hline TBAA & 3.90 & 4.30 & 4.64 & 6.15 & 5.66 & 6.17 & 6.84 & 7.33 & 5.54 & 5.84 & 5.30 & 4.90 \\
\hline
\end{tabular}

TEA: triethylamine; TBA: tributylamine; DBA: dibutylamine; DMBA: dimethyl- $n$-butylamine

than $300 \mathrm{mg} / \mathrm{L}$ and sufficient absolute recoveries (89-106\%) were obtained for all the iodoacetic acids [24]. In addition, the precursor and product ions of the anions (chlorate, bromate, iodate and perchlorate) in LC-MS/MS [36] are different from those of the HAAs in this study. Therefore, matrix interference from co-anions could be ignored. The occurrence and mean concentrations $(n=3)$ of the HAAs in finished water are listed in Table 5. All HAAs, especially
MIAA (concentration: $1.2 \pm 1.1 \mu \mathrm{g} / \mathrm{L})$, CIAA $(0.8 \pm 0.5 \mu \mathrm{g} / \mathrm{L})$ and BIAA $(0.3 \pm 0.2 \mu \mathrm{g} / \mathrm{L})$, were detected in the finished water from P1 in Shanghai. It should be noted that the concentrations of CIAA and BIAA were slightly lower than their LODs and not accurate because of limitation of the analytical method. More exact data will be obtained in further research. Iodoacetic acids were not detected in the finished water from P2 in Xuzhou. Formation of iodoacetic

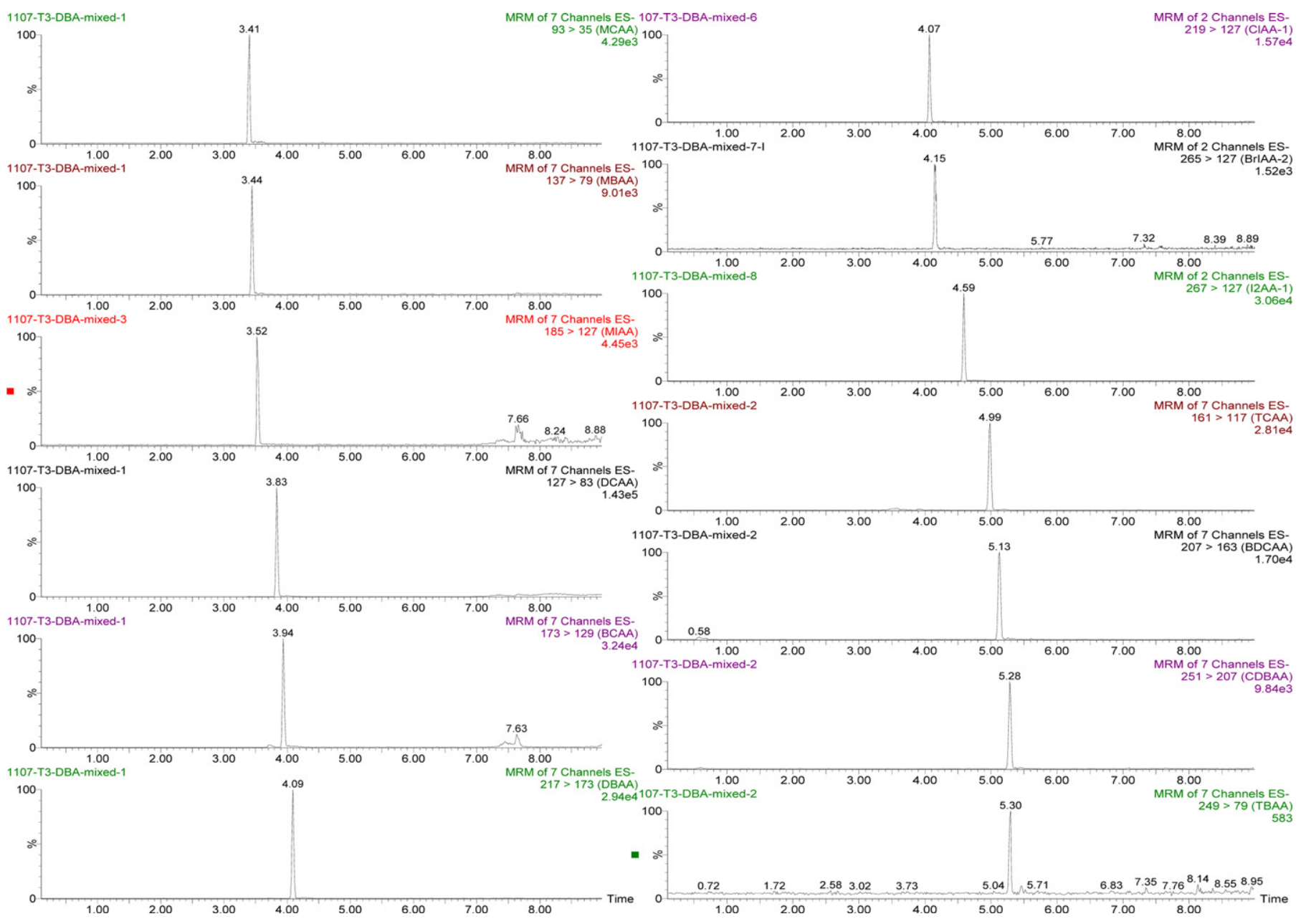

Fig. 1. Total ion chromatograms of the regulated and unregulated HAAs $(100 \mu \mathrm{g} / \mathrm{L})$ analyzed using a UPLC HSS C18 column with $5 \mathrm{mmol} / \mathrm{L}$ DBA as the ion pair reagent. 


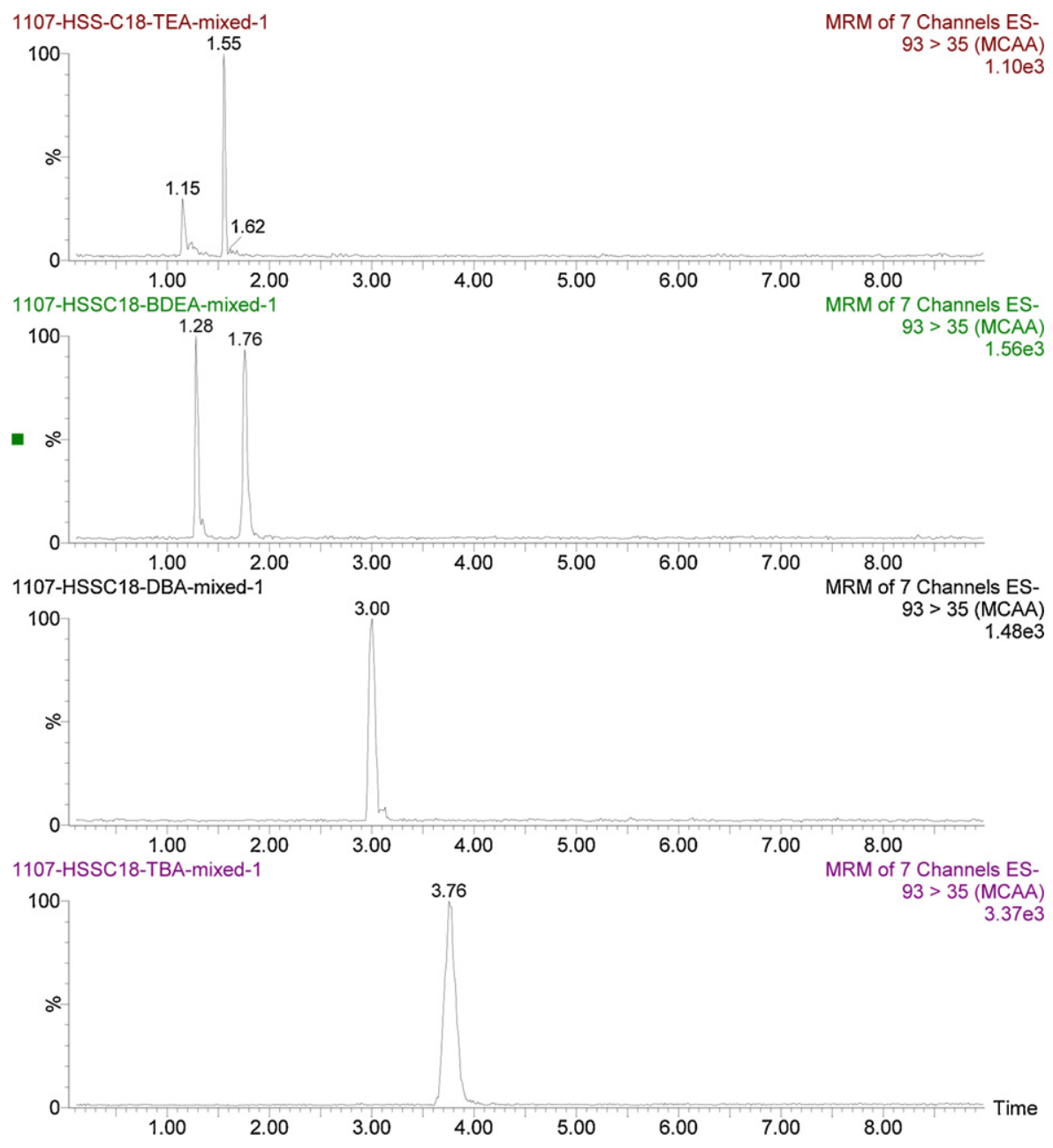

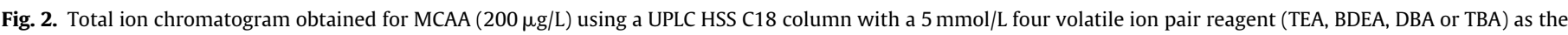
aqueous mobile phase.

acids is generally linked to the presence of chloramines. In a recent study, MIAA formation was detected when chlorinated tap water was boiled [31]. In the present study, iodoacids were found in the finished water from Shanghai, but not in that from Xuzhou. Because both water treatment stations use chlorine for disinfection, this difference could be attributed to the natural characteristics of the source water at each location.

The total HAA9 concentrations were 41.1 and $64.7 \mu \mathrm{g} / \mathrm{L}$ for P1 and $\mathrm{P} 2$, respectively. Among the HAAs, brominated and iodinated acetic acid concentrations in the finished water from Shanghai were higher than those in Xuzhou, although the total concentration of Shanghai was much less than in Xuzhou. This could be attributed to the natural characteristics of the source water. The sampling locations were representative of coastal (Shanghai) and inland (Xuzhou) cities in China. It is should be noted that up to $43.3 \%$ of the total HAAs concentration in the finished water from Xuzhou was MCAA, and its concentration was about two times that of DCAA and 10 times that of TCAA. The DCAA and TCAA concentrations in the finished water from Shanghai were $11.6 \pm 4.4$ and $5.3 \pm 7.2 \mu \mathrm{g} / \mathrm{L}$, respectively. By contrast, the concentrations of DCAA and TCAA in the Xuzhou sample were $15.1 \pm 8.7$ and $9.2 \pm 8.6 \mu \mathrm{g} / \mathrm{L}$, respectively. These concentrations are much lower

Table 4

Quality parameters of the method: linear relationship, limit of detection (LOD) and limit of quantitation (LOQ) for the 13 HAAs.

\begin{tabular}{|c|c|c|c|c|}
\hline HAAs & Regression equation & $r^{2}$ & $\operatorname{LOD}(\mu \mathrm{g} / \mathrm{L})$ & $\operatorname{LOQ}(\mu \mathrm{g} / \mathrm{L})$ \\
\hline MCAA & $Y=0.00249813^{*} X+0.0291549$ & 0.9999 & 0.35 & 1.6 \\
\hline MBAA & $Y=0.00311122 * X+0.0897743$ & 0.9976 & 0.15 & 0.5 \\
\hline MIAA & $Y=0.00469255^{*} X+0.0071234$ & 0.9881 & 0.45 & 1.5 \\
\hline DCAA & $Y=0.0241947^{*} X+0.734177$ & 0.9862 & 0.15 & 0.65 \\
\hline BCAA & $Y=0.0144368^{*} X+0.0472327$ & 0.9825 & 0.15 & 0.65 \\
\hline DBAA & $Y=0.00366383 * X+0.171192$ & 0.9896 & 0.35 & 1.7 \\
\hline CIAA & $Y=0.0644368^{*} X+0.0109232$ & 0.9711 & 0.45 & 2.1 \\
\hline BIAA & $Y=0.0414434^{*} X+0.0474243$ & 0.9740 & 0.45 & 2.1 \\
\hline DIAA & $Y=0.0094368^{*} X+0.0122327$ & 0.9844 & 0.5 & 2.5 \\
\hline TCAA & $Y=0.0220572 * X+0.0655029$ & 0.9821 & 0.25 & 1.2 \\
\hline BDCAA & $Y=0.0551045^{*} X+0.0352523$ & 0.9754 & 1.2 & 4.6 \\
\hline CDBAA & $Y=0.00984401 * X+0.0406903$ & 0.9744 & 1.5 & 5.8 \\
\hline TBAA & $Y=0.0277441^{*} X+0.134078$ & 0.9969 & 0.5 & 2.1 \\
\hline
\end{tabular}




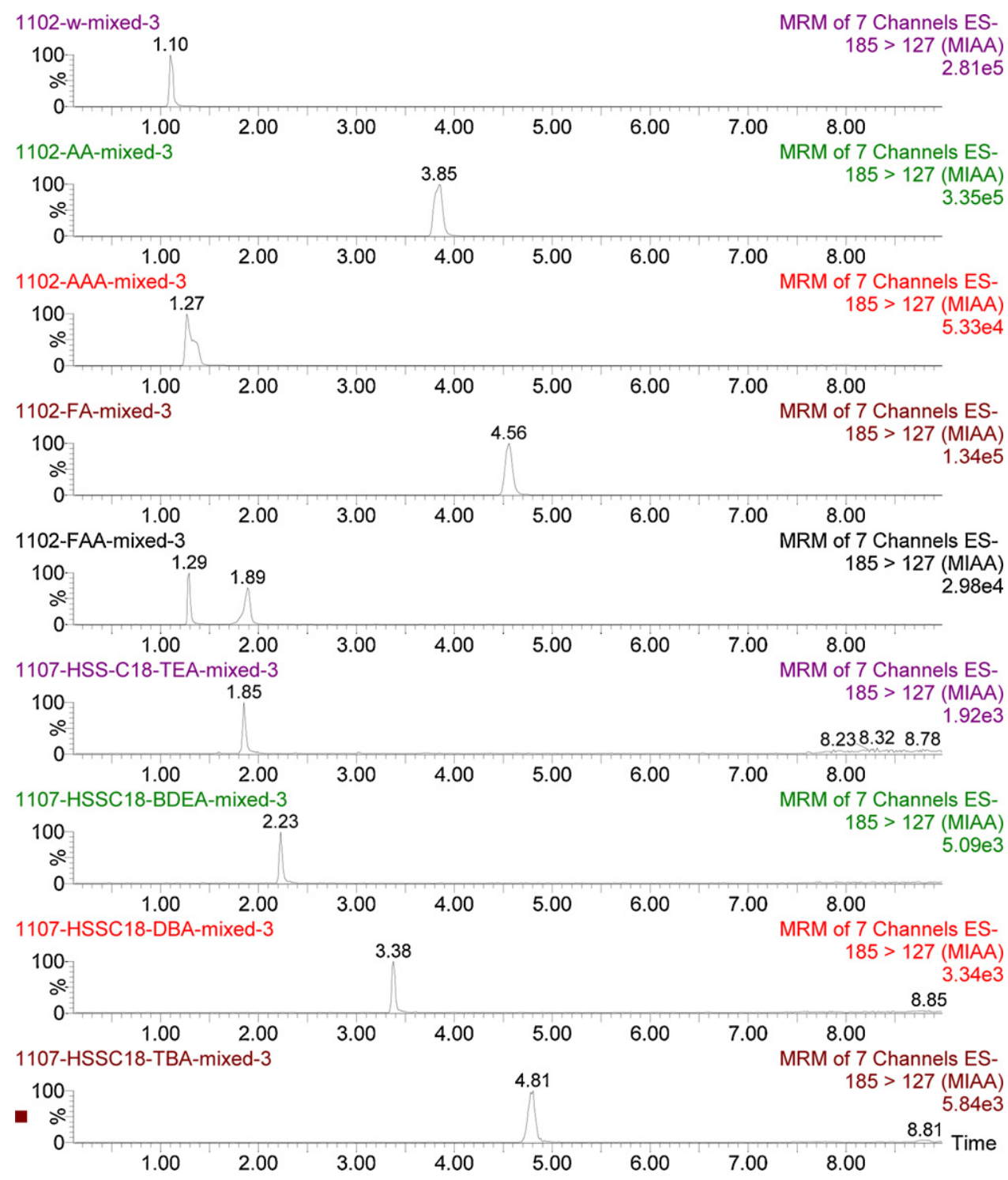

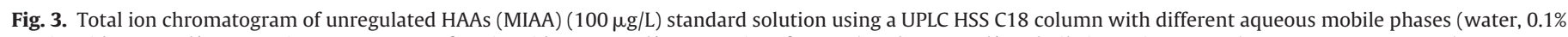
acetic acids, $5 \mathrm{mmol} / \mathrm{L}$ ammonium acetate, $0.1 \%$ formic acid, or $5 \mathrm{mmol} / \mathrm{L}$ ammonium formate) and a $5 \mathrm{mmol} / \mathrm{L}$ volatile ion pair reagent (TEA, BDEA, DBA or TBA).

Table 5

Occurrence and concentration levels ( $\mu \mathrm{g} / \mathrm{L}$ ) [mean $\pm \mathrm{RSD}(\%), n=3$ ] of HAAs in finished water samples from two drinking water treatment plants (P1 and P2) China.

\begin{tabular}{lll}
\hline & P1(Shanghai) & P2(Xuzhou) \\
\hline MCAA & $4.4 \pm 2.8$ & $28 \pm 9.7$ \\
MBAA & $8.2 \pm \pm 6.4$ & n.d. \\
MIAA & $1.2 \pm 1.1$ & n.d. \\
DCAA & $11.6 \pm 4.4$ & $15.1 \pm 8.7$ \\
BCAA & $0.9 \pm 0.2$ & $4.7 \pm 4.2$ \\
DBAA & $3.8 \pm 2.3$ & n.d. \\
CIAA & $0.8 \pm 0.5^{\mathrm{a}}$ & n.d. \\
BIAA & $0.3 \pm 0.2^{\mathrm{a}}$ & n.d. \\
DIAA & n.d. & n.d. \\
TCAA & $5.3 \pm 4.2$ & $9.2 \pm 8.6$ \\
BDCAA & $3.2 \pm 2.8$ & $4.1 \pm 3.1$ \\
CDBAA & $2.1 \pm 1.8$ & $2.9 \pm 2.1$ \\
TBAA & $1.6 \pm 0.9$ & $2.7 \pm 1.6$ \\
HAA5 & 28.9 & 47.8 \\
HAA9 & 41.1 & 64.7 \\
\hline
\end{tabular}

P: drinking water treatment plants; HAA5: sum of MCAA, DCAA, TCAA, MBAA and DBAA; HAA9: sum of MCAA, DCAA, TCAA, MBAA, DBAA, TBAA, BCAA, BDCAA and CDBAA.

a Data are not accurate, LOD < concentration level < LOQ. than the MCLs $(50 \mu \mathrm{g} / \mathrm{L}$ for DCAA and $100 \mu \mathrm{g} / \mathrm{L}$ for TCAA) [4,5]. The total concentrations of the HAA5 species in the Shanghai and Xuzhou samples were 28.9 and $47.8 \mu \mathrm{g} / \mathrm{L}$, respectively. These concentrations comply with the US EPA first stage regulations $(60 \mu \mathrm{g} / \mathrm{L})$, but the Xuzhou result slightly exceeds the second stage regulations [6]. The total concentration of HAA5 species was higher in the Xuzhou sample than the Shanghai sample. It was also higher than in tap water collected from Shangdong, China [9].

\section{Conclusion}

A simple and fast method is presented that is suitable for the analysis of HAAs in drinking water. In contrast to using conventional ion pair reagents as the aqueous mobile phase, the volatile amine DBA is added directly to each water sample can be used with MS. The optimized method was applied to investigate the distribution and concentrations of 13 HAAs in two finished water samples from water treatment plants in China. Chloro-, bromo-, and iodoacetic acids were found at different levels in these samples, and the relative concentrations could be attributed to the natural characteristics of the different source water and the disinfection method. 


\section{Acknowledgments}

This study was partially supported by grants from the National Natural Science Foundation of China (Grant Nos. NSFC21007077 and NSFC51290283) and the Ministry of Water Resources' Special Funds for Scientific Research on Public Causes (Grant No. 201201032).

\section{Appendix A. Supplementary data}

Supplementary data associated with this article can be found, in the online version, at http://dx.doi.org/10.1016/j.chroma. 2012.12.046.

\section{References}

[1] National Toxicology Program Clinical Evaluation Committee Draft Report. Arthur D. Little, Inc. Retrieved from http://ntp.niehs.nih.gov/? objectid= 03DADAD8-DFB8-5274-3E46A0CEA 167B37D

[2] US EPA Integrated Risk Information System. Dichloroacetic acid (CASRN 79-436). Retrieved from http://www.epa.gov/iris/subst/0654.htm

[3] US EPA Integrated Risk Information System. Trichloroacetic acid (CASRN 7603-9). Retrieved from http://www.epa.gov/ncea/iris/subst/0655.htm

[4] Revision of the World Health Organization (WHO) guidelines for drinking water quality, WHO, Geneva, 1991.

[5] Chinese National Standard GB 5749-2006, Standards for drinking water quality. Ministry of Health People's Republic of China.

[6] USEPA, National primary drinking water regulations: stage 2 disinfectants and disinfection byproducts rule, 2006. http://water.epa.gov/lawsregs/rulesregs/ sdwa/stage2/index.cfm

[7] Y.C. Hsu, Environ. Anal. 36 (2001) 22.

[8] S.W. Krasner, H.S. Weinberg, S.D. Richardson, S.J. Pastor, R. Chinn, M.J. Sclimenti, G.D. Onstad, A.D. Thruston Jr., Environ. Sci. Technol. 40 (2006) 7175.

[9] L. Meng, S. Wu, F. Ma, A. Jia, J. Hu, J. Chromatogr. A 1217 (2010) 4873.

[10] H.S. Weinberg, S.W. Krasner, S.D. Richardson, A.D. Thruston Jr. EPA/600/R02/068. U.S. Environmental Protection Agency, National Expo-sure Research Laboratory, Athens, GA, 2002.
[11] A. Becalski, B.P.Y. Lau, T.J. Schrader, S.W. Seaman, W.F. Sun, Food Addit. Contam. 23 (2006) 957.

[12] M.J. Plewa, E.D. Wagner, S.D. Richardson, A.D. Thruston Jr., Y.T. Woo, A.B. McKague, Environ. Sci. Technol. 15 (2004) 4713.

[13] S.D. Richardson, F. Fasano, J.J. Ellington, F.G. Crumley, K.M. Buettner, J.J. Evans, B.C. Blount, L.K. Silva, T.J. Waite, G.W. Luther, A.B. McKague, R.J. Miltner, E.D. Wagner, M.J. Plewa, Environ. Sci. Technol. 42 (2008) 8330.

[14] U. Bichsel, Gunten Von, Environ. Sci. Technol. 33 (1999) 4040

[15] S.E. Duirk, C. Lindell, C.C. Cornelison, J. Kormos, T.A. Ternes, M. Attene-Ramos, J Osiol, E.D. Wagner, M.J. Plewa, S.D. Richardson, Environ. Sci. Technol. 45 (2011) 6845.

[16] Standard method 6251B, disinfection by-products: haloacetic acids and trichlorophenol, micro liquid-liquid extraction GC method, in: A.D. Eaton, L.S Clesceri, A.E. Greenburg (Eds.), Standard Methods for the Examination of Water and Wastewater, 19th ed., 1995.

[17] L. Barron, B. Puall, Talanta 69 (2006) 621.

[18] M.C. Bruzzoniti, R.M. De Carlo, K. Horvath, D. Perrachon, A. Prelle, R. Tofalvi, C. Sarzanini, P. Hajos, J. Chromatogr. A 1187 (2008) 188

[19] A.M. Dixon, D.C. Delinsky, J.V. Bruckner, J.W. Fisher, M.G. Bartlett, J. Liq. Chromatogr. Relat. Technol. 27 (2004) 2343.

[20] A. Apfel, J.A. Chakel, S. Fischer, K. Lichtenwalte, W.S. Hancock, J. Chromatogr. A 777 (1997) 3.

[21] C.Y. Chen, S.N. Chang, J. Chromatogr. Sci. 47 (2009) 67.

[22] M. Takino, S. Daishima, K. Yamaguchi, Analyst 125 (2000) 1097.

[23] B.P.Y. Lau, A. Becalski, Rapid Commun. Mass Sp. 22 (2008) 1787.

[24] Y. Li, J.S. Whitaker, C.L. McCarty, J. Chromatogr. A 1245 (2012) 75.

[25] C. Rafols, D. Barcelo, J. Chromatogr. A 777 (1997) 177.

[26] C. Zwiener, S.D. Richardson, TrAC, Trend Anal. Chem. 24 (2005) 613.

[27] R. Roehl, R. Slingby, N. Avdolovic, P.E. Jackson, J. Chromatogr. A 956 (2002) 245

[28] J.M. Duan, W. Li, J. Si, D. Mulcahy, Anal. Methods-U.K. 3 (2011) 1667.

[29] D. Marínez, F. Borrull, M. Calull, M. Ruana, A. Colom, Chromatographia 49 (1998) 811.

[30] R. Loos, D. Barcelo, J. Chromatogr. A 938 (2001) 45.

[31] J. Kim, Environ. Res. 109 (2009) 158.

[32] S. Hashimoto, A. Otsuki, J. High Res. Chromatogr. 21 (1998) 55.

[33] M.S. Lee, Integrated Strategies for Drug Discovery Using Mass Spectrometry, John Wiley \& Sons Inc., New Jersey, 2000.

[34] L.R. Snyder, J.J. Kirkland, Introduction to Modern Liquid Chromatography, 2nd ed., John Wiley \& Sons Inc., New York, 1979.

[35] T. Storm, T. Reemtsma, M. Jekel, J. Chromatogr. A 854 (1999) 175.

[36] S.A. Snyder, B.J. Vanderford, D.J. Rexing, Environ. Sci. Technol. 39 (2005) 4586 